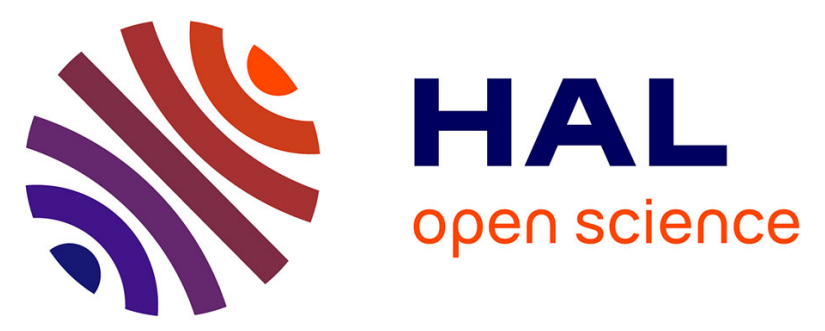

\title{
Analysis on the Impact of Different Time Constants of Smoothing Filter on Compliance of Ranging Source Monitors in Dual-Frequency GBAS \\ Junesol Song, Carl Milner
}

\section{- To cite this version:}

Junesol Song, Carl Milner. Analysis on the Impact of Different Time Constants of Smoothing Filter on Compliance of Ranging Source Monitors in Dual-Frequency GBAS. ION GNSS+ 2020, 33rd International Technical Meeting of the Satellite Division of the Institute of Navigation, Sep 2020, Virtual event, United States. pp. 1132-1143., 10.33012/2020.17580 . hal-02964006

\section{HAL Id: hal-02964006 \\ https://hal-enac.archives-ouvertes.fr/hal-02964006}

Submitted on 13 Oct 2020

HAL is a multi-disciplinary open access archive for the deposit and dissemination of scientific research documents, whether they are published or not. The documents may come from teaching and research institutions in France or abroad, or from public or private research centers.
L'archive ouverte pluridisciplinaire HAL, est destinée au dépôt et à la diffusion de documents scientifiques de niveau recherche, publiés ou non, émanant des établissements d'enseignement et de recherche français ou étrangers, des laboratoires publics ou privés. 


\title{
Analysis on the Impact of Different Time Constants of Smoothing Filter on Compliance of Ranging Source Monitors in Dual-Frequency GBAS
}

\author{
Junesol Song and Carl Milner, ENAC, Université de Toulouse, Frances
}

\section{BIOGRAPHY (IES)}

Junesol Song is a postdoctoral fellow in Telecom laboratory at the Ecole Nationale de l'Aviation Civile (ENAC) in Toulouse, France. She received B.S. and Ph.D. degrees in aerospace engineering from Seoul National University, South Korea. Her research interests include dual-frequency/multi-constellation GBAS fault monitoring, multi-GNSS Network RTK, Compact RTK, carrier phase-based algorithms and positioning, and remote sensing.

Dr. Carl Milner is an assistant professor within the Telecom Lab at the Ecole Nationale de l'Aviation Civile, Toulouse France. He currently lectures on many aspects of navigation science and technology including radio navigation aids, signal processing, positioning algorithms and GNSS for civil aviation. His research work addresses the design of integrity monitoring algorithms for current GNSS signals and the future multi-constellation multifrequency environment.

\begin{abstract}
In Ground Based Augmentation System (GBAS), the ground and the airborne uses the same magnitude of time constants for the carrier smoothing filter. This is to avoid the relative errors induced by the ionospheric divergence according to Minimal Operational Performance Standards (MOPS) airborne requirement [1]. This is especially true for a single-frequency GBAS solutions such as GBAS Approach Service Types -C and -D, which are to support the Category (CAT) I and CAT II/III precision approaches, respectively. However, in a dual-frequency and multi-constellation GBAS solution GAST-F, which is currently under development within the frame of Single European Sky ATM Research (SESAR) project, the ionospheric delay is completely eliminated through the Ionosphere-Free (IF) mode processing. Therefore, this might allow the airborne side to have flexible time constants of the carrier smoothing filter. In general, using larger time constant for a carrier smoothing filter is more effective in decreasing the standard deviation of the pseudorange measurements when the measurement error is assumed Gaussian. However, in reality, there exists a time-correlated error, namely a multipath error in the measurement, which limits the reduction of a standard deviation of the filtered measurement. Even with this, having the longer time constants would be beneficial to reduce the noise and multipath errors under the assumption that the mean of the multipath error is close to zero. Despite of having this merits of using larger time constant, it should be careful to change the time constant at the airborne, because the impact of the range error is related to the compliance of the monitor performance to the requirement, especially for the GBAS solutions that support CAT II/III [2]. In this paper, we analyze the impact of using nonhomogeneous time constants at the ground and the airborne, especially on the compliance of the ranging source monitor for the Code-Carrier Divergence (CCD) and for the signal deformation faults.
\end{abstract}

\section{INTRODUCTION}

The carrier smoothing filter is mandatory to mitigate the pseudorange noise and multipath error both at the ground and the airborne in a civil safety-critical system such as Satellite Based Augmentation System (SBAS) and Ground Based Augmentation System (GBAS). In general, the pseudorange noise can be reduced far more if a larger time constant is used for a carrier smoothing filter. However, it is not true for the single-frequency carrier smoothing filter because the performance of the filter is limited due to the divergence between pseudorange and carrier phase measurements. It should also be noted that the other factors such as multipath can 
also affect the filter performance, but we are focusing only on the divergence error in this study. The divergence is caused from having the opposite signs of ionospheric delays in pseudorange and carrier phase measurements. The impact of this divergence error in the filtered pseudorange measurement is proportional to the time constant of the carrier smoothing filter when the ionospheric delay is modeled as a ramp [3]. Consequently, the time constant needs to be selected carefully considering the performance of the smoothing of the pseudorange noise and the accumulation of the bias error due to the divergence error. Currently, for the singlefrequency GBAS solution, GBAS Approach Service Type (GAST)-C, which supports the Category (CAT) I precision approach, the ground and airborne smoothing filters use the same 100-second time constant. In GAST-D, which supports the CAT II/III precision approach for single-frequency, the 30 -second time constant is recommended by standards for ground and airborne smoothing filter. If the same time constants are used at ground and airborne, the remaining error due to the ionospheric divergence can be completely eliminated in a steady-state sense when the airborne pseudorange is corrected by applying the pseudorange correction from the ground receivers in a single-frequency processing.

The dual-frequency and multi-constellation GBAS solution which supports CAT II/III is defined as the GAST-F. The use of an additional frequency allows us to compute the Ionosphere-Free (IF) or Divergence-Free (DF) measurement combinations, consequently the carrier smoothing filter is not affected by the ionospheric delay anymore. The Minimum Operational Performance Standards (MOPS) recommends matching the time constants at the ground and airborne to avoid the relative error induced by the divergence error [1]. Therefore, more freedom could be given when determining the time constant of carrier smoothing filter in dualfrequency processing mode, such as the GAST-F.

This study assesses the validity of using nonhomogeneous time constants used at ground and airborne smoothing filter in terms of the ranging source monitors. This is based on the fact that the compliance of the monitor to the anticipated GAST-F requirements depends not only on the monitor performance but also on the differential range error between the ground and the airborne [2]. To prevent the airborne receivers from misleading information (MI) due to ranging source faults, such as the Code-Carrier Divergence (CCD) and the signal deformation faults, ranging source monitors are mandatory at the ground receiver. The GAST-D provides requirements of the Probability of Missed Detection (PMD) for the monitors to detect ranging source faults [2] and the same requirement may be applied to GAST-F subject to caveats regarding the processing mode. This requirement relates the differential range error and the PMD of the monitor. The differential range error is caused by ranging source faults and it depends on the time constants of the carrier smoothing filters at the ground and the airborne. Consequently, the PMD compliance of the monitor with the requirement can be affected by the different time constants used at the ground and airborne smoothing filter. Therefore, in this study, we investigate the PMD compliance of the CCD and signal deformation monitors with the requirement according to different time constants of the carrier smoothing filter at the ground and airborne.

In the first part of the paper, the impact of the CCD and signal deformation faults in the range error is modeled as a ramp and a step error, respectively. These impacts in range domain are derived as analytical equations to see the transient and steady-state behaviors for various time constants used at the ground and the airborne smoothing filters.

In the second part of the paper, the simulation for the assessment of the PMD compliance of the ranging source monitor is conducted according to different time constants used at the airborne carrier smoothing filter while the time constant at the ground is fixed to 100 seconds. Previously, the ENAC has proposed the dual-frequency CCD monitor to protect airborne users against the dualfrequency CCD faults [4]. In addition, the combination of the conventional Honeywell Signal Quality Monitor (SQM) [5], ENAC DF-Innovation monitor [6] and ENAC CCD monitors have been proposed to deal with the signal deformation fault. Using these solutions for the CCD and signal deformation faults, the compliance of the PMD is determined for various time constants at the airborne carrier smoothing filter.

In the simulation, firstly, the CCD faults and the signal deformation faults are generated and added in the pseudorange measurement. Specifically, the signal deformation faults are generated on a chip level with respect to ICAO standards for GPS L1 signal and some recommendations which are currently under discussion for the standardization for Galileo E1 and E5 signals [7]. The generated signal distortion is then added to the GNSS code sequence and the correlation function is computed. After applying the pre-correlation filter, the code-tracking offset is computed by inspecting the filtered correlation function with the Early-Late correlator. The associated range error can be computed by comparing the chip offsets of the distorted and nominal signals. The theoretical standard deviation of the correlator outputs, which has been validated by a real data collection at ENAC using iFen Software Receiver, are computed for determining the threshold of Honeywell SQM. Global Positioning System (GPS) L1/L5 and Galileo E1/E5 signals are collected from the GBAS research-oriented prototype to obtain the statistics of the CCD and DF-Innovation monitors.

In this assessment, the various airborne filter initialization times are also taken into account, because the differential range error can be varied according to relative timings of the ground and airborne filter initialization times and the fault onset time even for the same fault cases. The nominal case can be that the both filters at ground and airborne are initialized far earlier than the fault onset time. The worst case for the PMD compliance occurs when the airborne smoothing is initialized just after the fault onset time. In this case 
the differential range error becomes large before the fault is fully incorporated in the monitor. In this case the pseudorange smoothing filter at the ground is initialized far earlier which is guaranteed by ground subsystem wait times specified in the standards. Consequently, the PMD compliance was tested also for various filter initialization times of the airborne receiver.

The result can provide the information on the impact of the various time constants in the aspect of the PMD compliance of the ranging source monitors within the dual-frequency and the multi-constellation GBAS solution, GAST-F. It also can be taken into account for the future recommendation on the time constant for the carrier smoothing filter at the ground and the airborne.

\section{CARRIER SMOOTHING FILTER}

The block diagram of the carrier smoothing filter is shown in Figure 1 and the possible inputs for the symbols $\Psi$ and $\Phi$ in Figure 1 are represented in Table 1 according to various processing modes. The symbols of $\rho$ and $\phi$ are pseudorange and carrier phase, respectively. The constant $\gamma$ indicates the squared ratio of the L1 and L5 frequencies. The equation for the output of the smoothing filter in Laplace domain can be expressed as the following equation.

$$
\begin{aligned}
y(s) & =F(s) e_{\Psi}(s)+(1-F(s)) e_{\Phi}(s) \\
& =\frac{1}{\tau s+1} e_{\Psi}(s)+\frac{\tau s}{\tau s+1} e_{\Phi}(s)
\end{aligned}
$$

The constant $\tau$ indicates the time constant of the smoothing filter, and the terms $e_{\psi}$ and $e_{\Phi}$ are the impact of the ranging source faults on pseudorange and carrier phase measurements, respectively. After some further manipulations of the equation (1), it can be expressed as the differential equation in time domain as shown below.

$$
\frac{d}{d t}\left\{e^{\frac{t}{\tau}} y(t)\right\}=e^{\frac{t}{\tau}} \dot{e}_{\Phi}(t)+\frac{1}{\tau} e^{\frac{t}{\tau}} e_{\Psi}(t)
$$

In the next section, the steady state differential range error will be analyzed according to the CCD and signal deformation faults.

Table 1 Inputs for carrier smoothing filter according to various processing modes

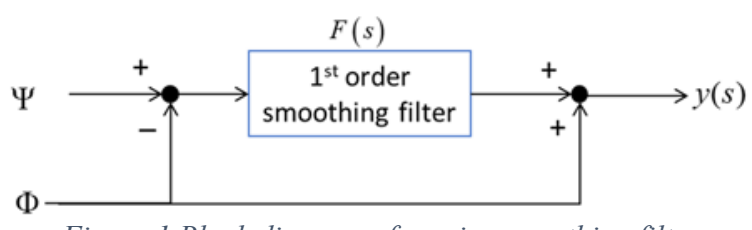

\begin{tabular}{|c|c|c|}
\hline Processing Modes & $\boldsymbol{\Psi}$ & $\boldsymbol{\Phi}$ \\
\hline $\begin{array}{c}\text { L1 single } \\
\text { frequency }\end{array}$ & $\rho_{L 1}$ & $\phi_{L 1}$ \\
\hline $\begin{array}{c}\text { L5 single } \\
\text { frequency }\end{array}$ & $\rho_{L 5}$ & $\phi_{L 5}$ \\
\hline L1 DF & $\rho_{L 1}$ & $\phi_{L 1}+2 \frac{\phi_{L 1}-\phi_{L 5}}{\gamma-1}$ \\
\hline L5 DF & $\rho_{L 5}$ & $\phi_{L 5}+2 \gamma \frac{\phi_{L 1}-\phi_{L 5}}{\gamma-1}$ \\
\hline IF & $\frac{\gamma \rho_{L 1}-\rho_{L 5}}{\gamma-1}$ & $\frac{\gamma \phi_{L 1}-\phi_{L 5}}{\gamma-1}$ \\
\hline
\end{tabular}

\section{THEORETICAL ANALYSIS OF THE IMPACT OF USING NONHOMOGENEOUS TIME CONSTANTS}

The CCD fault and the signal deformation fault can be modeled as ramp and step errors respectively in range domain [8-9]. If we assume that the ranging source fault is occurred at $t_{f}$, the impact of the CCD and signal deformation faults in range, denoted as $e_{\Psi}$ and $e_{\Phi}$, can be defined as the following equations.

$$
\text { - For CCD fault, } \begin{aligned}
e_{\Psi}(t) & = \begin{cases}0 & t<t_{f} \\
d_{\Psi} \cdot\left(t-t_{f}\right) & t \geq t_{f}\end{cases} \\
e_{\Phi}(t) & = \begin{cases}0 & t<t_{f} \\
d_{\Phi} \cdot\left(t-t_{f}\right) & t \geq t_{f}\end{cases}
\end{aligned}
$$




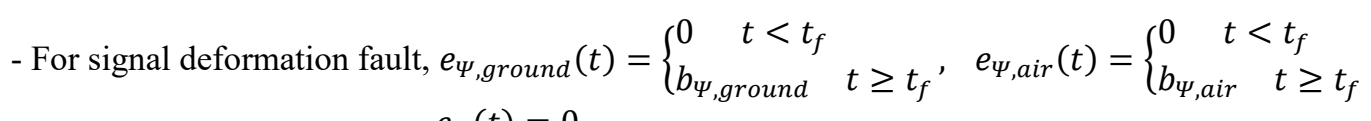

The symbols of $d_{\Psi}$ and $d_{\Phi}$ indicate the CCD rates in pseudorange and carrier phase measurement with units of $\mathrm{m} / \mathrm{s}$. The terms $b_{\Psi, \text { ground }}$ and $b_{\Psi, \text { air }}$ are the impacts of the signal deformation faults on pseudoranges at the ground and the airborne, respectively. It should be noted that the impact of the fault should be discriminated at ground and airborne for the signal deformation case. This is because the impact of the signal deformation fault on the range error depends on the receiver configurations unlike the CCD fault. Using these definitions of the impact of the faults, the steady state differential range error can be derived in the following subsections.

\section{For CCD Faults}

From definition of the faults as in Equation (3) and (4), the range error due to the faults at ground and airborne equals to zero for $t<t_{f}$. For $t>t_{f}$, the Equation (2) can be expressed as the following equation.

$$
e_{\text {ground }}(t)=d_{\Psi} \cdot\left[\left(t-\tau_{g}-t_{f}\right)+\tau_{g} e^{-\frac{t-t_{f}}{\tau_{g}}}\right]+d_{\Phi} \cdot \tau_{g}\left(1-e^{-\frac{t_{f}}{\tau_{g}}}\right)
$$

To distinguish smoothing time constants at the ground and the airborne, the subscript ' $\mathrm{g}$ ' has been added to the symbol, $\tau$. In this derivation, we assumed that the ground filter has been initialized far earlier than the time of fault onset. The Pseudo-Range Correction (PRC) generated at the ground includes the impact of the CCD fault expressed as Equation (5). However, it should be noted that the PRC that the airborne uses at the time, $t$ is not exactly the same as the Equation (5). This is because of the correction broadcast interval and the ground processing time. For GAST-F, these two terms are generally set to 2.0 seconds and 1 seconds, respectively [10]. The comprehensive term is defined as $t_{\text {gdelay }}$ and the definition of the rate of the PRC and the estimated PRC used at the airborne can be defined as the following equations.

$$
\begin{aligned}
\dot{e}_{\text {ground }}(t) & =d_{\Psi} \cdot\left(1-e^{-\frac{t-t_{f}}{\tau_{g}}}\right)+d_{\Phi} \cdot e^{-\frac{t_{f}}{\tau_{g}}} \\
\hat{e}_{\text {ground }}(t) & =e_{\text {ground }}\left(t-t_{\text {gdelay }}\right)+\dot{e}_{\text {ground }}\left(t-t_{\text {gdelay }}\right) \cdot t_{\text {gdelay }} \\
& =d_{\Psi} \cdot\left\{t-\tau_{g}-t_{f}+\left(\tau_{g}-t_{\text {gdelay }}\right) e^{-\frac{t_{\text {gdelay }}-t_{f}}{\tau_{g}}}\right\}+d_{\Phi} \cdot\left\{\tau_{g}+\left(t_{\text {gdelay }}-\tau_{g}\right) e^{-\frac{t-t_{\text {gdelay }}-t_{f}}{\tau_{g}}}\right\}
\end{aligned}
$$

At the airborne, there are two possible derivations of the range error according to the relative times of the airborne filter initialization time $\left(t_{a}\right)$ to the fault onset as the following equations.

$$
\begin{aligned}
& \text { - For } t_{a}<t_{f}: \quad e_{a i r}(t)=d_{\Psi} \cdot\left[\left(t-\tau_{a}-t_{f}\right)+\tau_{a} e^{-\frac{t-t_{f}}{\tau_{a}}}\right]+d_{\Phi} \cdot \tau_{a}\left(1-e^{-\frac{t-t_{f}}{\tau_{a}}}\right) \\
& \text { - For } t_{a} \geq t_{f}: \quad e_{a i r}(t)=d_{\Psi} \cdot\left[\left(t-\tau_{a}-t_{f}\right)+\left(\tau_{a}+t_{a}-t_{f}\right) e^{-\frac{t-t_{a}}{\tau_{a}}}\right]+d_{\Phi} \cdot \tau_{a}\left(1-e^{-\frac{t-t_{a}}{\tau_{a}}}\right)
\end{aligned}
$$

Accordingly, the differential range error, denoted as $E_{R}(t)$, between the ground and the airborne can be expressed as following according to the timings of $t_{a}$.

$$
\text { - For } t_{a}<t_{f}: E_{R}(t)= \begin{cases}e_{\text {air }}(t) & t_{f} \leq t \leq t_{f}+t_{\text {gdelay }} \\ -\left(d_{\Psi}-d_{\Phi}\right) \cdot\left\{\tau_{a}\left(1-e^{-\frac{t-t_{a}}{\tau_{a}}}\right)-\tau_{g}\left(1-e^{-\frac{t-t_{\text {gdelay }}-t_{f}}{\tau_{g}}}\right)-t_{\text {gdelay }} \cdot e^{-\frac{t-t_{\text {gdelay }}-t_{f}}{\tau_{g}}}\right\} \quad t \geq t_{f}+t_{\text {gdelay }}\end{cases}
$$




$$
\text { - For } t_{a} \geq t_{f}: E_{R}(t)=\left\{\begin{array}{l}
e_{\text {air }}(t) \quad t_{f} \leq t \leq t_{f}+t_{\text {gdelay }} \\
e^{-\frac{t-t_{a}}{\tau_{a}}} \cdot d_{\psi} \cdot\left(t_{a}-t_{f}\right)-\left(d_{\psi}-d_{\Phi}\right) \cdot\left\{\tau_{a}\left(1-e^{-\frac{t-t_{a}}{\tau_{a}}}\right)-\tau_{g}\left(1-e^{-\frac{t-t_{\text {gdelay }}-t_{f}}{\tau_{g}}}\right)-t_{\text {gdelay }} \cdot e^{-\frac{t-t_{\text {gdelay }}-t_{f}}{\tau_{g}}}\right\} \quad t \geq t_{f}+t_{\text {gdelay }}
\end{array}\right.
$$

For both cases, the differential range error approaches to $\left(d_{\Psi}-d_{\Phi}\right) \cdot\left(\tau_{g}-\tau_{a}\right)$ at the steady state. That is, if the nonhomogeneous time constants are being used at the ground and the airborne, non-zero steady state error remains, which is proportional to the difference of time constants at the ground and the airborne. This can be problematic if the PMD of the CCD monitor is less than $10^{-}$ 5 , which will be addressed in detail in the following section. The time history of the differential range errors for possible timings of $t_{a}$ are shown in Figure 2 for various values of time constants at the airborne. The simulated CCD faults for $d_{\Psi}$ and $d_{\Phi}$ are $0.088 \mathrm{~m} / \mathrm{s}$ and $-0.018 \mathrm{~m} / \mathrm{s}$, respectively. In this simulation, the time constant at the ground is fixed to 100 seconds. Because the airborne filter is implemented as a time variant filter, each curve has the same profile for 30/ $t$ t epochs. For both cases of (a) and (b), the differential range error approaches to zero only when the homogeneous time constants are used at the ground and the airborne. It's clearly observed that the steady-state error is proportional to the difference of time constants used at the ground and the airborne.

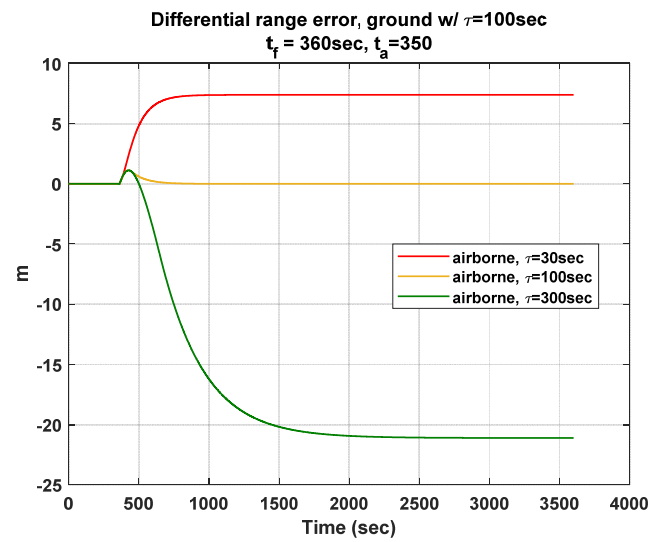

(a)

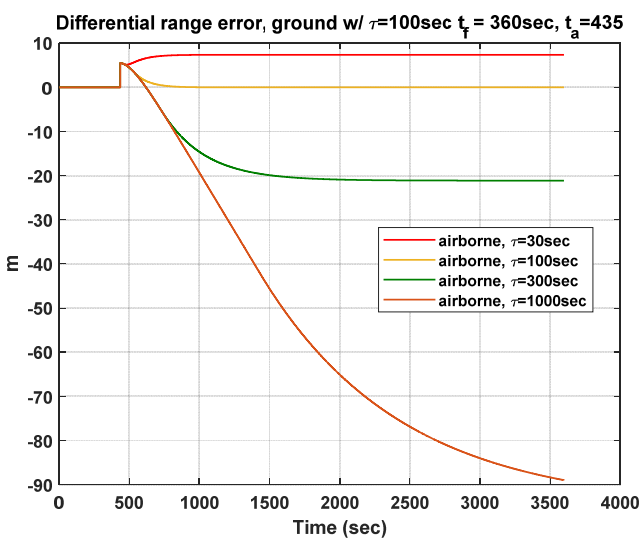

(b)

Figure 2 Time history of the differential range error due to the CCD faults when a fixed time constant is used at the ground (100 seconds) for various time constants at the airborne, (a) $t_{a}<t_{f}$, and (b) $t_{a} \geq t_{f}$ cases

\section{For Signal Deformation Faults}

The derivation procedure is similar to that for the CCD faults. The equations for the range error at the ground and airborne, and the differential range error are shown as the following equations.

- Range error at the ground: $e_{\text {ground }}(t)=b_{\Psi, \text { ground }} \cdot\left(1-e^{-\frac{t-t_{f}}{\tau_{g}}}\right)$

- Range error in the PRC which is used at the airborne: $\hat{e}_{\text {ground }}(t)=b_{\Psi, \text { ground }} \cdot\left\{1-\left(1-\frac{t_{\text {gdelay }}}{\tau_{g}}\right) e^{-\frac{t-t_{\text {gdelay }}-t_{f}}{\tau_{g}}}\right\}$

- Range error at the airborne

$$
\begin{aligned}
& \text { - For } t_{a}<t_{f}: e_{\text {air }}(t)=b_{\Psi, \text { air }} \cdot\left(1-e^{-\frac{t-t_{f}}{\tau_{a}}}\right) \\
& \text { - For } t_{a} \geq t_{f}: e_{\text {air }}(t)=b_{\Psi, \text { air }}
\end{aligned}
$$

- Differential range error

$$
\begin{aligned}
& \text { - For } t_{a}<t_{f}: E_{R}(t)=\left\{\begin{array}{l}
e_{\text {air }}(t) \quad t_{f} \leq t \leq t_{f}+t_{\text {gdelay }} \\
b_{\Psi, \text { air }} \cdot\left(1-e^{-\frac{t-t_{f}}{\tau_{a}}}\right)-b_{\Psi, \text { ground }} \cdot\left\{1-\left(1-\frac{t_{\text {gdelay }}}{\tau_{g}}\right) e^{-\frac{t-t_{\text {gdelay }}-t_{f}}{\tau_{g}}}\right\} t \geq t_{f}+t_{\text {gdelay }}
\end{array}\right. \\
& \text { - For } t_{a} \geq t_{f}: E_{R}(t)=\left\{\begin{array}{l}
e_{\text {air }}(t) \quad t_{f} \leq t \leq t_{f}+t_{\text {gdelay }} \\
b_{\Psi, \text { air }}-b_{\Psi, \text { ground }} \cdot\left\{1-\left(1-\frac{t_{\text {gdelay }}}{\tau_{g}}\right) e^{-\frac{t-t_{\text {gdelay }}-t_{f}}{\tau_{g}}}\right\} t \geq t_{f}+t_{\text {gdelay }}
\end{array}\right.
\end{aligned}
$$


For possible timings of $t_{a}$, the differential range error approaches to $b_{\Psi, \text { air }}-b_{\Psi, \text { ground }}$ at the steady state. Therefore, using nonhomogeneous time constants at the ground and the airborne does not impact on the differential range error. Figure 3 shows the time history of differential range error for (a) $t_{a}<t_{f}$, and (c) $t_{a} \geq t_{f}$ cases when $b_{\Psi, \text { ground }}$ and $b_{\Psi, \text { air }}$ are set to $0.88 \mathrm{~m}$ and $0.4 \mathrm{~m}$, respectively. Figure 3(b) is for $t_{a}<t_{f}$ case, but with different magnitude of the faults: $b_{\Psi, \text { ground }}=-0.4 \mathrm{~m}$ and $b_{\Psi, \text { air }}=0.88 \mathrm{~m}$. In this simulation, the fault is assumed to be occurred at 360 seconds $\left(t_{f}=360\right)$. The simulation confirms that the steady-state error is independent on the homogeneity or non-homogeneity of the time constants at the ground and the airborne. It should be noted, however, that the profiles of the transient period can be affected by the non-homogeneity of the time constant as shown in Figure $3(\mathrm{a})$ and (b). It needs to be checked through simulation if it affects the compliance of the monitor to the requirement. For the case that the airborne filter is initialized after the fault onset, the time histories of the differential range errors are the same for all values of the time constants, which validates the derived Equation (17). In Figure 3(a) and (c), the sudden increase occurred right after the fault onset for (a) and right after the airborne filter initialized for (b) is due to the ground delay which causes few epochs of period that the impact of the fault is not incorporated in correction while the airborne measurement already has its impact.

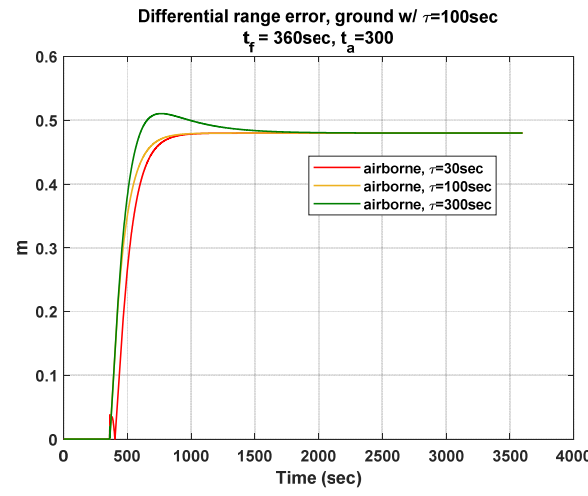

(a)

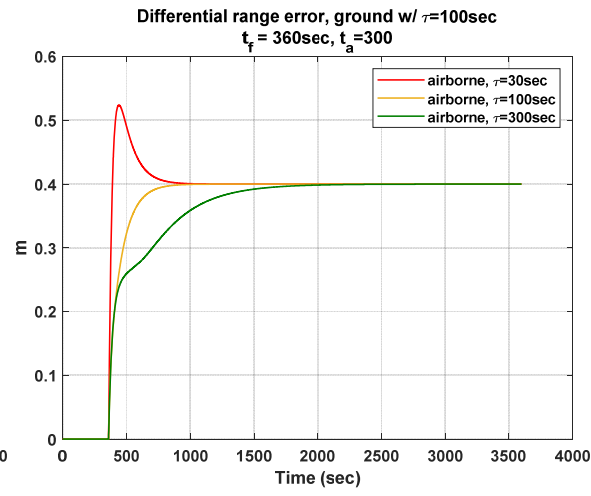

(b)

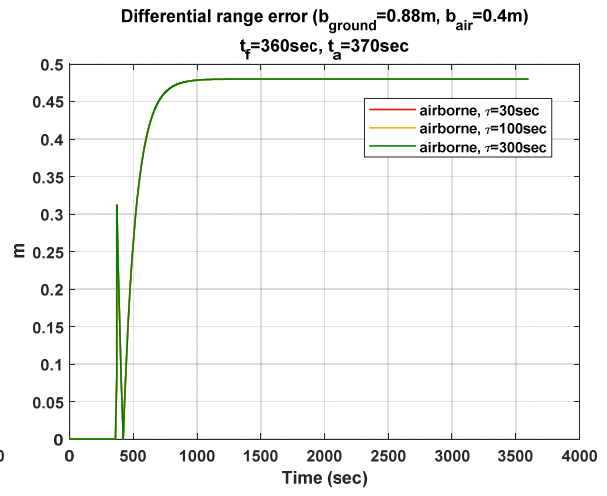

(c)

Figure 3 Time history of the differential range error due to the signal deformation faults when a fixed time constant is used at the ground (100 seconds) for various time constants at the airborne, $(a, b) t_{a}<t_{f}$, and (c) $t_{a} \geq t_{f}$ cases. For (a) and (c), $b_{\Psi, \text { ground }}$ and $b_{\Psi, \text { air }}$ are set to $0.88 \mathrm{~m}$ and $0.4 \mathrm{~m}$. For $(b)$, they are set to $-0.4 \mathrm{~m}$ and $0.88 \mathrm{~m}$, respectively.

\section{REQUIREMENT ON RANGING SOURCE MONITOR FOR GAST-D AND GAST-F}

The requirement on the PMD of the ranging source fault monitor is defined in the standards for GAST-D [2]. This defines the allowable limit values of the PMD according to the absolute of differential range error as shown in Figure 4, which has been derived from the consideration of limit case and malfunction case. The requirement for GAST-F can be more relaxed because of the improved satellite geometry due to the use of a multi-constellation. However, the requirement specific for GAST-F has not been defined yet in the standards as well as for the conservative analysis, the same requirement for GAST-D will be used for GAST-F in this assessment.

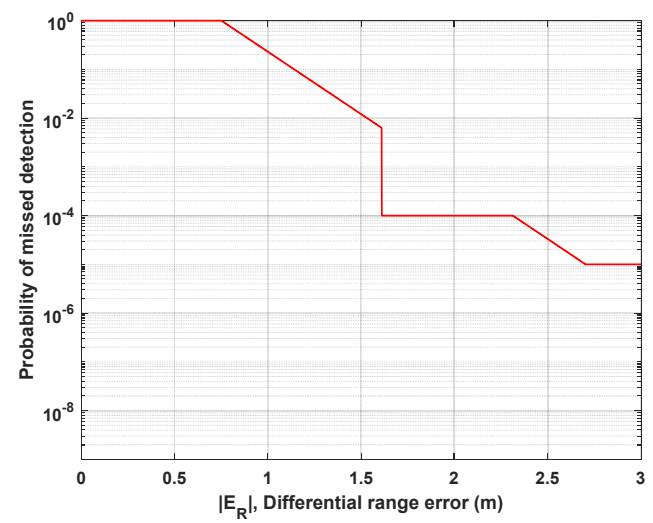

Figure 4 PMD constraints according to differential range error 


\section{SIMULATION CONFIGURATION}

In the assessment for the CCD fault, the dual-frequency chi-square CCD monitor [4], which combines the rate of the DF1 and DF5 Code Minus Carrier (CMC) and the IF carrier phase, is adopted. In [4], the CCD fault is considered separately on each frequency and pseudorange/carrier phase. The threat space of the CCD rate on L1/L5 pseudorange is ranged from $-0.025 \mathrm{~m} / \mathrm{s}$ to $+0.025 \mathrm{~m} / \mathrm{s}$ with $0.0025 \mathrm{~m} / \mathrm{s}$ interval. And for the L1/L5 carrier phase, it is set from $-0.005 \mathrm{~m} / \mathrm{s}$ to $+0.005 \mathrm{~m} / \mathrm{s}$ with $0.0005 \mathrm{~m} / \mathrm{s}$ interval according to [4]. As stated in [6], the magnitude of the maximum differential range error depends on the relative time $\left(t_{a}-t_{f}\right)$ of the airborne filter initialization time $\left(t_{a}\right)$ to the fault onset time $\left(t_{f}\right)$. Therefore, the assessment is conducted for various values for the airborne filter initialization time as shown in Table 2.

For the signal deformation fault, the SQM solution proposed in [6], which uses the ENAC CCD monitor [4], Honeywell SQM [5] and ENAC DF-Innovation monitor [6], is adopted. The signal distortion is generated in chip-level for GPS L1 C/A signal according to the ICAO standards [2]. For Galileo E1 and E5 signals, the recent proposition of the threat space [7] for the standardization is used to generate the distortion. The digital, analog and their combinations, namely Threat Model (TM) A, B and C are taken into account. For the airborne initialization time, 50 seconds and 360 seconds are tested to take into account for the consideration of the impact of the relative time between $t_{a}$ and $t_{f}$ on differential range error. Because the signal deformation fault causes a step error in pseudorange, only two extreme cases for $t_{a}<t_{f}$ and $t_{a} \geq t_{f}$ are tested.

To fully mimic the GAST-F system, the design parameters such as latencies of the correction and integrity messages and Time-ToAlert recommended for GAST-F are considered as shown in Table 2 below. In addition, as recommended in [12], we used the term $t_{\text {delay }}$, which indicates the intentional time delay after the airborne filter initialization before incorporating measurements for navigation, to help satisfying the requirement especially for $t_{a} \geq t_{f}$ case.

\section{Table 2 Simulation configuration}

\begin{tabular}{|c|c|c|}
\hline \multicolumn{2}{|c|}{ Simulation parameters } & Values \\
\hline \multicolumn{2}{|c|}{ Latency of correction message $\left(\tau_{G}\right)$} & $3 \mathrm{sec}$ (message update interval 2.0sec + ground delay $1 \mathrm{sec}$ ) [10-11] \\
\hline \multicolumn{2}{|c|}{ Latency of Integrity message $\left(\tau_{I}\right)$} & $1.5 \mathrm{sec}$ (message update interval $0.5 \mathrm{sec}+$ ground delay $1 \mathrm{sec})[11]$ \\
\hline \multicolumn{2}{|c|}{$\begin{array}{c}\text { Time-To-Detect and Affect the } \\
\text { BroadcAst (TTDABA) }\end{array}$} & $1.5 \mathrm{sec}(0.5 \mathrm{sec}$ missed integrity messages $+1.0 \mathrm{sec}$ margin $)[3,11]$ \\
\hline \multirow{2}{*}{$\begin{array}{l}\text { Time constant for } \\
\text { carrier smoothing }\end{array}$} & Ground $\left(\tau_{\text {ground }}\right)$ & $100 \mathrm{sec}$ \\
\hline & Airborne $\left(\tau_{\text {air }}\right)$ & 30,100 and $300 \mathrm{sec}$ \\
\hline \multicolumn{2}{|c|}{ Time of fault onset $\left(t_{f}\right)$} & $360 \mathrm{sec}$ \\
\hline \multicolumn{2}{|c|}{ Filter initialization time at ground $\left(t_{g}\right)$} & $0 \mathrm{sec}$ \\
\hline \multicolumn{2}{|c|}{$\begin{array}{l}\text { Filter initialization time at airborne } \\
\qquad\left(t_{a}\right)\end{array}$} & $\begin{array}{l}\text { - Test for CCD: } 300 \mathrm{sec} \text { to } 1400 \mathrm{sec} \text { with } 10 \text {-seconds interval } \\
\text { - Test for signal deformation fault: } 50 \mathrm{sec} \text { and } 360 \mathrm{sec}\end{array}$ \\
\hline \multicolumn{2}{|c|}{$\begin{array}{l}\text { Airborne time delay for incorporating } \\
\text { measurement for navigation }\left(t_{\text {delay }}\right)\end{array}$} & For the case of $t_{a} \geq t_{f}, 0$ to 132 seconds of $t_{\text {delay }}$ are tested \\
\hline
\end{tabular}

\section{SIMULATION RESULTS}

The PMD compliance results for the CCD faults are shown in Figure 5. The plots in each row show the results for different values for $\tau_{\text {air }}$ such as 30,100 and 300 seconds. The plots in the left and right columns indicate the cases for different values of $t_{\text {delay }}, 50$ and 132 seconds, respectively according to [4]. The smoothing time constant at the airborne is tested for 30 (Figure 5(a) and (b)), 100 (Figure 5(c) and (d)) and 300 (Figure 5(e) and (f)) seconds.

In Figure 5(c) and (d), which represent the results for the homogeneous smoothing time constants at the ground and the airborne, the monitor satisfies the requirement if the $t_{\text {delay }}$ is increased to 132 seconds. However, for the nonhomogeneous smoothing time constants are used at the ground and the airborne, the monitor is not compliant to the requirement even for larger $t_{\text {delay }}$. As pointed out previously, this is because of the non-zero steady-state differential range error which is proportional to the difference between time constants at the ground and the airborne. 


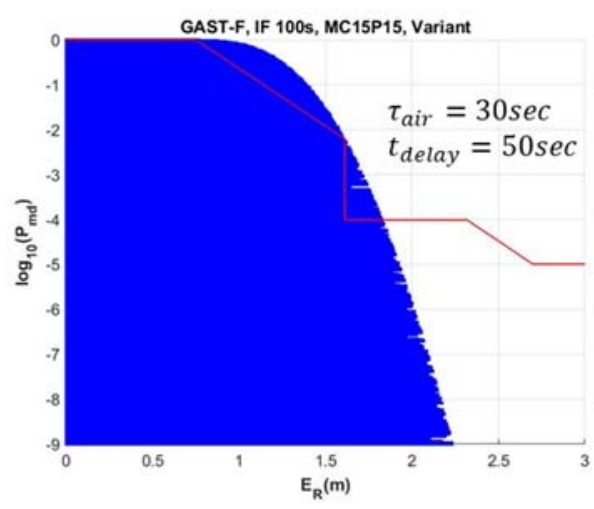

(a)

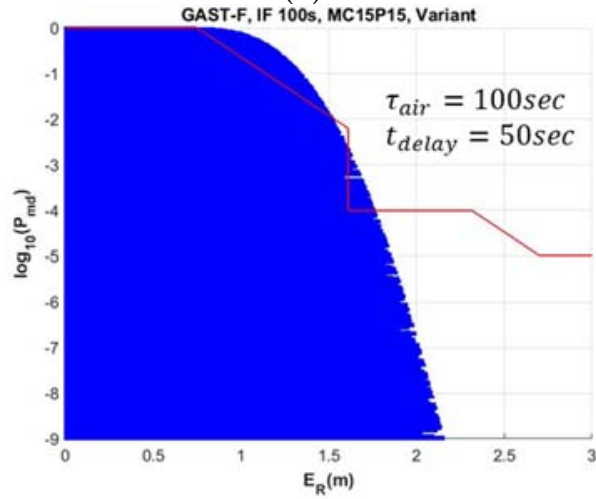

(c)

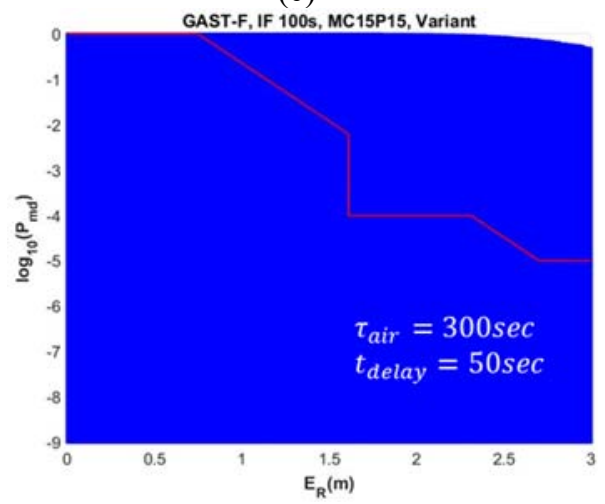

(e)

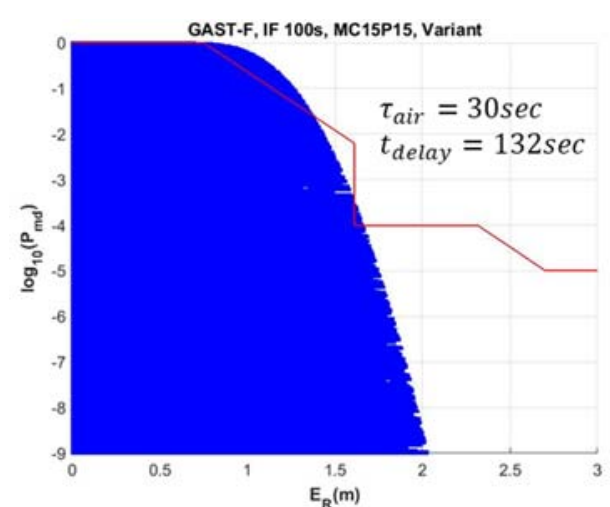

(b)

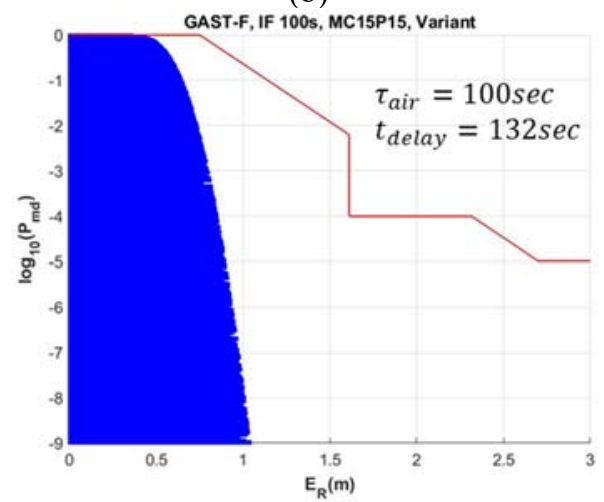

(d)

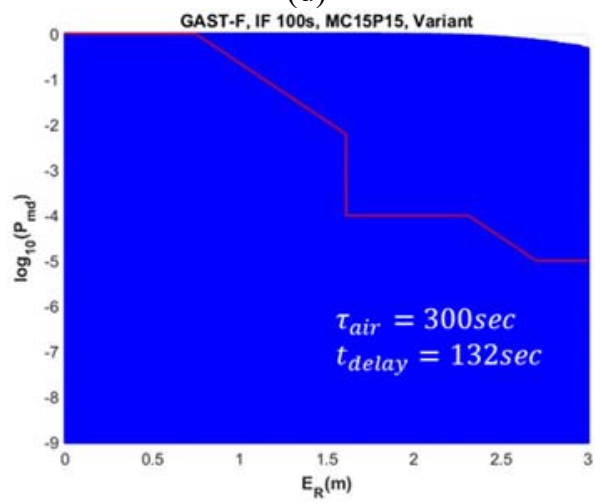

(f)

Figure 5 The PMD compliance results for CCD faults for various airborne smoothing time constants

For a specific CCD fault, the performance of the monitor reaches to some level as shown in Figure 6(a). However, unlike the case that uses the homogeneous time constants, the differential range error reaches to the non-zero steady-state error when nonhomogeneous time constants are involved, so the monitor performance might not be good enough for larger differential range error to satisfy the requirement. In Figure 6(c), the monitor performance is not enough for the case when $\tau_{\text {air }}$ is set to 300 seconds. This is because the larger the differential range error is, the smaller PMD is required. Since $t_{\text {delay }}$ is effective in removing epochs right after the airborne filter initialization as shown in Figure 6(d), for which the monitor performance is vulnerable to satisfy the requirement, from the performance assessment, it does not help nonhomogeneous cases to satisfy the requirement as in Figure 5(b) and (f). 


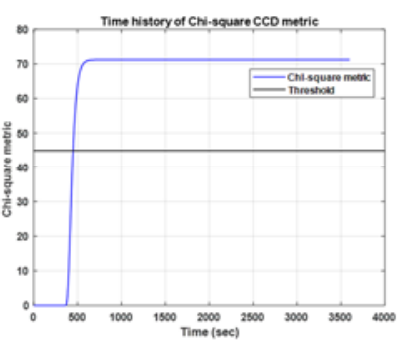

(a)

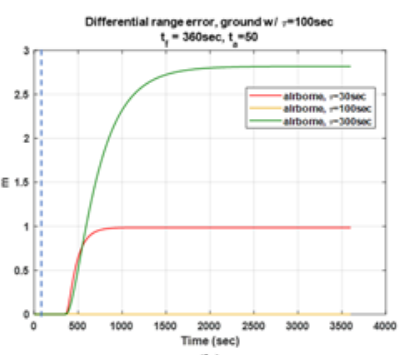

(b)

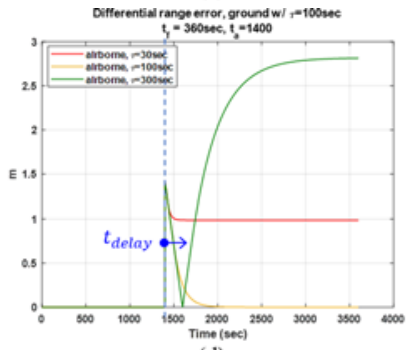

(d)

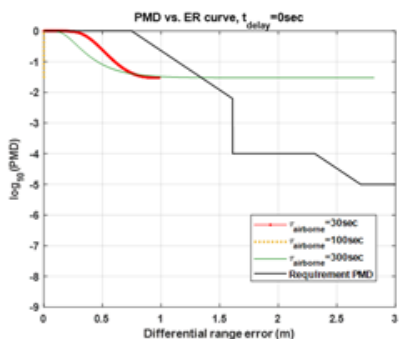

(c)

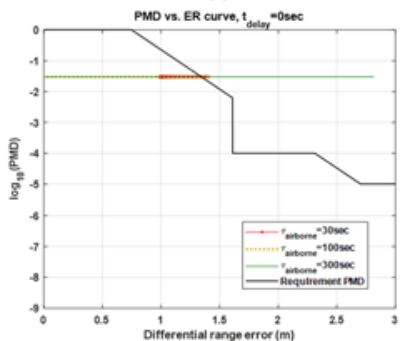

(e)

Figure 6 (a) Time history of the CCD monitor and its threshold, $(b, c)$ Time history of the differential range error and the PMD vs. ER requirement curve for $t_{a}<t_{f}$, and $(d, e)$ Time history of the differential range error and the PMD $v$. ER requirement curve for $t_{a} \geq t_{f}$. The vertical dashed blue line indicates the $t_{a}$, and $d_{\Psi}$ and $d_{\Phi}$ are set to $-0.01 \mathrm{~m} / \mathrm{s}$ and $-0.002 \mathrm{~m} / \mathrm{s}$, respectively.

Because the noncompliant cases occur for non-zero steady-state error of the differential range error, which is caused by the difference between time constants at the ground and airborne, we can consider using an additional monitor at the ground which detects a fault in the difference of two filtered IF pseudorange with different time constants. This reminds us a Dual Smoothing Ionospheric Gradient Monitoring Algorithm (DSIGMA) [13] used at the airborne. To check if the range-domain DSIGMA at the ground modified for the IF combination can potentially solve the problem, this monitor is tested for a single CCD fault case to which the existing CCD monitor is not compliant. For this test, we assumed that the DSIGMA uses the difference of two filtered IF pseudorange with 100second and 300-second time constants. The standard deviation of the monitor is computed using data collection from the Multipath Limiting Antenna (MLA) of the GBAS research-oriented prototype developed within the frame of SESAR proejct with 0.5 second interval on May $8^{\text {th }}, 2019$. The over-bounded standard deviation of the monitor was approximately $0.11 \mathrm{~m}$. In order to compute the threshold, the probability of fault free alarm for the CCD fault, $1.5 \times 10^{-7}$ per $15 \mathrm{sec}$ [8] is divided by half and allocated for each monitor, the existing CCD monitor and the range-domain IF DSIGMA at the ground. The $d_{\Psi}$ and $d_{\Phi}$ are set to $0.0048 \mathrm{~m} / \mathrm{s}$ and 0 $\mathrm{m} / \mathrm{s}$, respectively.

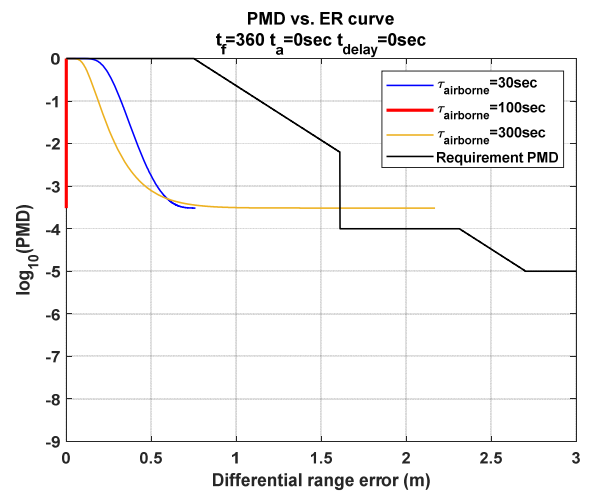

(a)

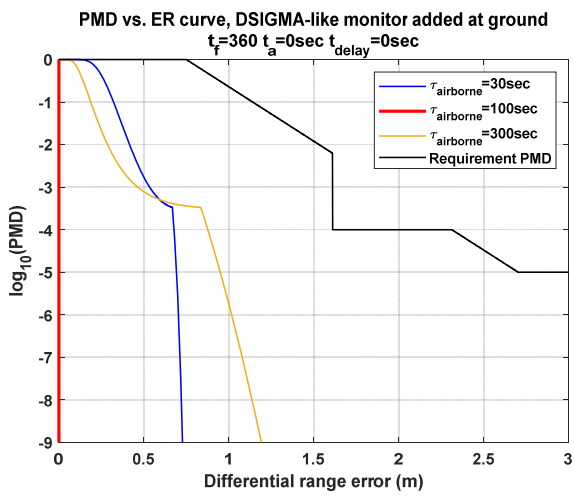

(b)

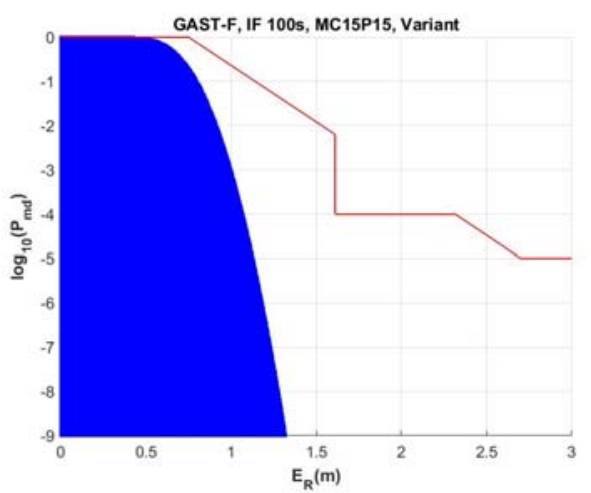

(c)

Figure 7 The PMD vs. ER curve for (a) only the existing CCD monitor is used, and (b,c) the existing CCD monitor and range-domain IF DSIGMA (100 and 300-second time constants) are used. The subplot (c) shows the compliance results for the full CCD threat space with coarse interval for $\tau_{\text {air }}=300 \mathrm{sec}$ and $t_{\text {delay }}=132 \mathrm{sec}$. The CCD rates $d_{\Psi}$ and $d_{\Phi}$ are set to $0.0048 \mathrm{~m} / \mathrm{s}$ and $0 \mathrm{~m} / \mathrm{s}$, respectively. 
Figure 7 shows the PMD versus differential range curve to determine the compliance of the monitors. Figure 7(a) is the result when only the ENAC CCD monitor is used, and Figure 7(b) and (c) show the results when the range-domain DSIGMA is added. Because the DSIGMA directly monitors the difference of two filtered IF pseudorange with different time constants of the smoothing filter, it can protect against the impact of the CCD faults on the steady-state differential range error with small probability of missed detection. Figure 7(c) indicates the results for the full CCD threat space with coarse interval when $\tau_{\text {air }}$ and $t_{\text {delay }}$ are set to $300 \mathrm{sec}$ and $132 \mathrm{sec}$, respectively. Based on this result, it is verified that the addition of the range-domain IF DSIGMA leads to compliance of the monitor for the increased airborne filter time constant.

Table 3 shows the compliance result for signal deformation faults. Each table shows the results for $\tau_{\text {air }}$ equals to 30,100 and 300 seconds. The green shade indicates the monitor satisfies the requirement and the red shade indicates the noncompliant results. Except for the case of $t_{a}<t_{f}$ in GPS L1 and Galileo E1c signals, the proposed SQM solution in [6] can successfully protect the GBAS system and user against the signal deformation faults which are within the threat space. For GPS L5 and Galileo E5a signal, we can observe that the required $t_{\text {delay }}$ is smaller than those for GPS L1 and Galileo E1c. In addition, Galileo E1c requires smaller $t_{\text {delay }}$ than the values that GPS L1 requires. We can infer from this observation that the signal structure of Galileo E1c is more robust against the signal distortion than that of GPS L1. We have noted from Figure 3(a) that using nonhomogeneous time constants is able to increase the magnitude of the differential range error during transient period. From Table 3, we can conclude that it does not impact on the compliance of the monitor. Figure 8 shows the impact of using different time constants at the airborne on the PMD vs. ER curves for GPS L1 and Galileo E1c signals.

Table 3 Compliance results for all signals and various fault scenarios. The green shade indicates the compliance and the red shade means the non-compliance. The required $t_{\text {delay }}$ for the compliance is indicated for $t_{a} \geq t_{f}$ case.

\begin{tabular}{|c|c|c|c|c|c|}
\hline \multicolumn{2}{|c|}{$\tau_{a i r}=30$ sec } & GPS L1 & GPS L5 & Galileo E1c & Galileo E5a \\
\hline \multirow{2}{*}{ TMA } & $\boldsymbol{t}_{a}<\boldsymbol{t}_{\boldsymbol{f}}$ & & & & \\
\cline { 2 - 6 } & $\boldsymbol{t}_{a} \geq \boldsymbol{t}_{\boldsymbol{f}}$ & $t_{\text {delay }}=0 \mathrm{sec}$ & $t_{\text {delay }}=0 \mathrm{sec}$ & $t_{\text {delay }}=0 \mathrm{sec}$ & $t_{\text {delay }}=0 \mathrm{sec}$ \\
\hline \multirow{2}{*}{$\mathrm{TMB}$} & $\boldsymbol{t}_{a}<\boldsymbol{t}_{\boldsymbol{f}}$ & & & & \\
\cline { 2 - 6 } & $\boldsymbol{t}_{a} \geq \boldsymbol{t}_{\boldsymbol{f}}$ & $t_{\text {delay }}=30 \mathrm{sec}$ & $t_{\text {delay }}=0 \mathrm{sec}$ & $t_{\text {delay }}=20 \mathrm{sec}$ & $t_{\text {delay }}=0 \mathrm{sec}$ \\
\hline \multirow{2}{*}{$\mathrm{TMC}$} & $\boldsymbol{t}_{a}<\boldsymbol{t}_{\boldsymbol{f}}$ & & & & \\
\cline { 2 - 6 } & $\boldsymbol{t}_{a} \geq \boldsymbol{t}_{\boldsymbol{f}}$ & $t_{\text {delay }}=20 \mathrm{sec}$ & $t_{\text {delay }}=10 \mathrm{sec}$ & $t_{\text {delay }}=0 \mathrm{sec}$ & $t_{\text {delay }}=10 \mathrm{sec}$ \\
\hline
\end{tabular}

\begin{tabular}{|c|c|c|c|c|c|}
\hline \multicolumn{2}{|c|}{$\tau_{a i r}=\mathbf{1 0 0}$ sec } & GPS L1 & GPS L5 & Galileo E1c & Galileo E5a \\
\hline \multirow{2}{*}{ TMA } & $\boldsymbol{t}_{a}<\boldsymbol{t}_{\boldsymbol{f}}$ & & & & \\
\cline { 2 - 6 } & $\boldsymbol{t}_{a} \geq \boldsymbol{t}_{\boldsymbol{f}}$ & $t_{\text {delay }}=0 \mathrm{sec}$ & $t_{\text {delay }}=0 \mathrm{sec}$ & $t_{\text {delay }}=0 \mathrm{sec}$ & $t_{\text {delay }}=0 \mathrm{sec}$ \\
\hline \multirow{2}{*}{$\mathrm{TMB}$} & $\boldsymbol{t}_{\boldsymbol{a}}<\boldsymbol{t}_{\boldsymbol{f}}$ & & & & \\
\cline { 2 - 6 } & $\boldsymbol{t}_{\boldsymbol{a}} \geq \boldsymbol{t}_{\boldsymbol{f}}$ & $t_{\text {delay }}=30 \mathrm{sec}$ & $t_{\text {delay }}=0 \mathrm{sec}$ & $t_{\text {delay }}=20 \mathrm{sec}$ & $t_{\text {delay }}=0 \mathrm{sec}$ \\
\hline \multirow{2}{*}{$\mathrm{TMC}$} & $\boldsymbol{t}_{\boldsymbol{a}}<\boldsymbol{t}_{\boldsymbol{f}}$ & & & & \\
\cline { 2 - 6 } & $\boldsymbol{t}_{\boldsymbol{a}} \geq \boldsymbol{t}_{\boldsymbol{f}}$ & $t_{\text {delay }}=20 \mathrm{sec}$ & $t_{\text {delay }}=10 \mathrm{sec}$ & $t_{\text {delay }}=0 \mathrm{sec}$ & $t_{\text {delay }}=10 \mathrm{sec}$ \\
\hline
\end{tabular}

\begin{tabular}{|c|c|c|c|c|c|}
\hline \multicolumn{2}{|c|}{$\tau_{\text {air }}=\mathbf{3 0 0}$ sec } & GPS L1 & GPS L5 & Galileo E1c & Galileo E5a \\
\hline \multirow{2}{*}{ TMA } & $\boldsymbol{t}_{a}<\boldsymbol{t}_{\boldsymbol{f}}$ & & & & \\
\cline { 2 - 6 } & $\boldsymbol{t}_{a} \geq \boldsymbol{t}_{\boldsymbol{f}}$ & $t_{\text {delay }}=0 \mathrm{sec}$ & $t_{\text {delay }}=0 \mathrm{sec}$ & $t_{\text {delay }}=0 \mathrm{sec}$ & $t_{\text {delay }}=0 \mathrm{sec}$ \\
\hline \multirow{2}{*}{ TMB } & $\boldsymbol{t}_{a}<\boldsymbol{t}_{\boldsymbol{f}}$ & & & & \\
\cline { 2 - 6 } & $\boldsymbol{t}_{a} \geq \boldsymbol{t}_{\boldsymbol{f}}$ & $t_{\text {delay }}=30 \mathrm{sec}$ & $t_{\text {delay }}=0 \mathrm{sec}$ & $t_{\text {delay }}=20 \mathrm{sec}$ & $t_{\text {delay }}=0 \mathrm{sec}$ \\
\hline \multirow{2}{*}{ TMC } & $\boldsymbol{t}_{a}<\boldsymbol{t}_{\boldsymbol{f}}$ & & & & \\
\cline { 2 - 6 } & $\boldsymbol{t}_{a} \geq \boldsymbol{t}_{\boldsymbol{f}}$ & $t_{\text {delay }}=20 \mathrm{sec}$ & $t_{\text {delay }}=10 \mathrm{sec}$ & $t_{\text {delay }}=0 \mathrm{sec}$ & $t_{\text {delay }}=10 \mathrm{sec}$ \\
\hline
\end{tabular}




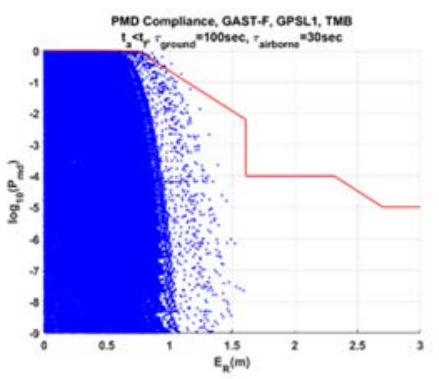

(a)

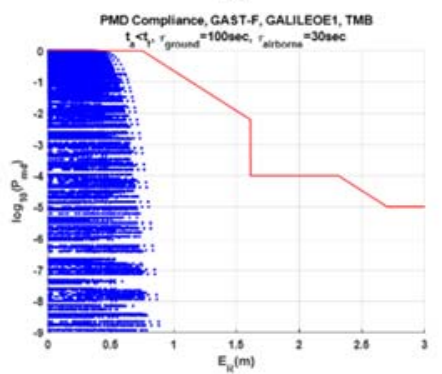

(d)

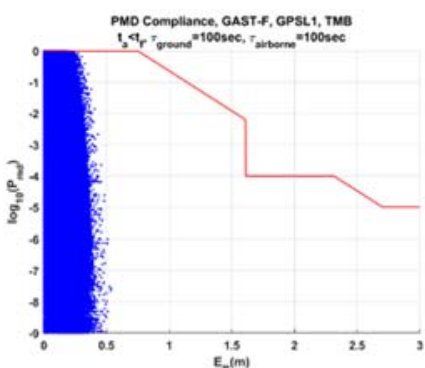

(b)

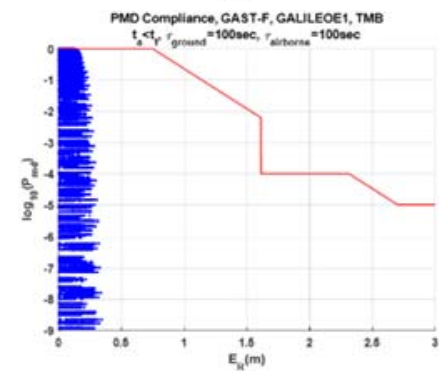

(e)

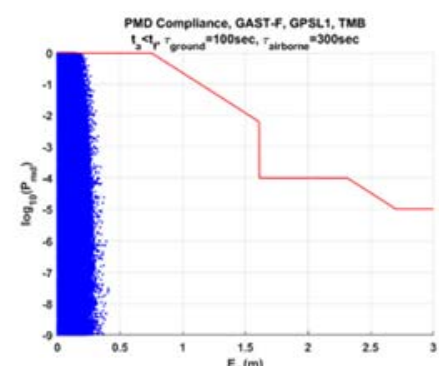

(c)

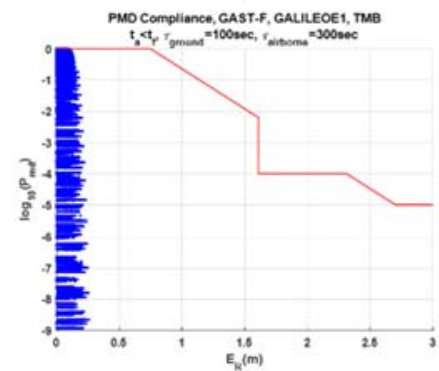

(f)

Figure 8 Example of PMD vs. ER curves of (a-c) GPS L1, and (d-f) Galileo E1c for TM-B when $t_{a}<t_{f}$.

\section{CONCLUSIONS}

In this paper, the impact of the nonhomogeneous time constants at the ground and the airborne as been analyzed in terms of the monitor performance. Use of a longer smoothing time constant is advantageous to improve the navigation performance, because ideally it can reduce the noise and multipath errors in pseudorange measurement more than when the shorter smoothing time constants is used. The time constants also affect the response of the range errors in the smoothing filter that are caused by ranging source faults such as the CCD and the signal deformation faults. Since the monitor requirement for the GAST-D and GAST-F depends not only on the PMD of the monitor but also on the differential range error, the selection of the smoothing time constant could also affect the monitor compliance to the requirement. In this assessment, we focus on the CCD and signal deformation faults to see the impact of the use of nonhomogeneous smoothing time constants at the ground and the airborne. Previously proposed monitors are used to assess the monitor performance.

For CCD faults, the use of nonhomogeneous smoothing time constants introduces a non-zero steady-state error in differential range errors unlike the case when the homogeneous time constants are used at the ground and the airborne. As a result, it limits the CCD monitor to satisfy the requirement for all fault cases within the threat space. The potential of using the range-domain IF DSIGMA to protect against the impact of the CCD fault on the steady-state differential range error has been investigated. This monitor is slightly modified from the existing DSIGMA. Using the statistics obtained from the research-oriented GBAS prototype equipped with the MLA, the result has shown that the addition of the range-domain IF DSIGMA at the ground can potentially solve the problem of the noncompliant cases occurred for the non-zero steady-state differential range errors. The performance evaluation for the full CCD threat space is left for our future work.

For signal deformation faults, the differential range error at the steady state is not affected by using the nonhomogeneous smoothing time constants. However, the value during the transient period might be increased and it is checked if this impacts on the compliance of the monitor to the requirement. As a result, the use of a shorter time constant at the airborne than at the ground limits the monitor to satisfy the requirement for $t_{a}<t_{f}$ case. On the other hand, in the opposite case, when a longer time constant is used at the airborne, it is observed that the monitor still can satisfy the requirement. Therefore, for signal deformation faults, it does not pose any problem in using a longer smoothing time constants at the airborne than that at the ground.

In this study, we have investigated the possibility of using nonhomogeneous time constants of the carrier smoothing filter at the ground and the airborne for the dual-frequency and the multi-constellation GBAS solution, GAST F. In conclusions, for signal deformation fault, the homogeneity does not pose any additional problem in terms of the monitor compliance. On the other hand, for the CCD fault, the noncompliant cases occurred due to the non-zero steady-state differential range error. However, we saw the potential to resolve this problem by adopting the range-domain IF DSIGMA at the ground. In conclusions, with an addition of a 
proper monitor, such as the range-domain IF DSIGMA at the ground, the use of larger time constants at the airborne can be considered in dual-frequency GBAS.

\section{ACKNOWLEDGMENTS}

This project has received funding from the SESAR Joint Undertaking under the European Union's Horizon 2020 research and innovation programme under grant agreement No 874478 .

Disclaimer: The opinions expressed herein reflect the author's view only. Under no circumstances shall the SESAR Joint Undertaking be responsible for any use that may be made of the information contained herein.

\section{REFERENCES}

1. DO-253C "Minimum Operational Performance Standards for the Local Area Augmentation System Airborne Equipment", RTCA, December 16, 2008.

2. ICAO SARPs (2018) Annex 10, Aeronautical Telecommunications.

3. Hwang, P.Y., McGraw, G. A., Bader, J. R., "Enhanced differential GPS carrier-smoothed code processing using dual-frequency measurements," Navigation, Vol. 46, No. 2, 1999, pp.127-138.

4. Jiang, Y., Milner, C. and Macabiau, C. "Code carrier divergence monitoring for dual-frequency GBAS", GPS Solutions, Springer Verlag, 2017, 21 (2), pp. Pages 769-781.

5. Liu, F., Brenner, M., Tang, C.Y., "Signal Deformation Monitoring Scheme Implemented in a Prototype Local Area Augmentation System Ground Installation," Proceedings of the 19th International Technical Meeting of the Satellite Division of The Institute of Navigation (ION GNSS 2006), Fort Worth, TX, September 2006, pp. 367-380.

6. Song, J., Milner, C., Selmi, "Signal deformation fault monitors for dual-frequency GBAS," Navigation, Vol. 67, No. 2, 2020, pp. 379-396.

7. Selmi, I., Thevenon, P., Macabiau, C., Julien, O., and Mabilleau, M., "Signal Quality Monitoring Algorithm Applied to Galileo Signals for Large Evil Waveform Threat Space," Proceedings of the 2020 International Technical Meeting of The Institute of Navigation, San Diego, California, Feb. 2020, pp. 352-365.

8. Simili, D. V., and Pervan, B., "Code-Carrier Divergence Monitoring for the GPS Local Area Augmentation System," Proceedings of 2006 IEEE/ION Position, Location, And Navigation Symposium, Coronado, CA, 25-27 April, pp. 483-493.

9. Phelts, E., "Multicorrelator techniques for robust mitigation of threats to GPS signal quality," Stanford University, 2001.

10. SESAR, D3.7.1 Monitoring, Integrity and Performance, 03.00.00, November 2015.

11. Milner, C., Guilbert, A., Macabiau, C. "Evolution of Corrections Processing for the MC/MF Ground Based Augmentation System (GBAS)," Proceedings of the 2015 International Technical Meeting of The Institute of Navigation, Dana Point, California, January 2015, pp. 364-373.

12. Brenner, M., Liu, F., "Ranging Source Fault Detection Performance for Category III GBAS," Proceedings of the 23rd International Technical Meeting of the Satellite Division of The Institute of Navigation (ION GNSS 2010), Portland, OR, September 2010, pp. 2618-2632.

13. Shively, C. A., Hsiao, T. T., "Availability of GAST D GBAS Considering Continuity of Airborne Monitors," Proceedings of the 2010 International Technical Meeting of The Institute of Navigation, San Diego, CA, January 2010, pp. 365-375. 